\title{
BLOCKING CELIAC PLEXUS WITH BOTULINUM TOXIN IN THE TREATMENT OF PAIN FOR CHRONIC PANCREATITIS - CASE REPORT
}

\author{
V.C. Paiva1 ${ }^{1}$, C.S. Roxo ${ }^{1}$, K.L.L. Fonseca ${ }^{1}$, H.S. Fernandes ${ }^{1}$, H.A. Ashmawi ${ }^{1}$, E.B. Espada ${ }^{1}$ \\ 1 Department of Pain and Anaesthesiology from Hospital das Clínicas - University of Sao Paulo - HCFMUSP - São Paulo (Brazil)
}

BACKGROUND: The main initial complaint of chronic pancreatitis is continuous and deep pain in epigastric region, frequently with irradiation to the back, which initially may be intermittent, but it progresses with time. The pain can be triggered by the ingestion of any food, especially the fatty ones and nauseas and vomits are common complaints, and there may be dehydration, malnutrition and the inability to take analgesics oral.

CASE REPORT: K. H. S., 47 years old, female, with complaint of abdominal pain due to chronic pancreatitis by hypertriglyceridemia. Visceral abdominal pain in hypogastrium, right hypochondrium, and left and right iliac fossa. Using for the treatment of pain: Tramadol 200mg/day, Methadone 15mg/day when needed, Gabapentin 1200mg/day, Amitriptyline $25 \mathrm{mg} /$ day. The patient was submitted on $04 / 24 / 17$ to a bilateral celiac plexus block with total injection of $40 \mathrm{ml}$ of Ropivacaine $0.25 \% \quad(100 \mathrm{mg})$ and $200 \mathrm{IU}$ of botulinum toxin, guided by ultrasonography. The average pre-procedure pain was $7 / 10$ (numerical verbal scale), ranging from $6 / 10$ to $8 / 10$. Pain immediately after procedure was $0 / 10$, a with improvement of $100 \%$. In a telephone interview 3 months after, the patient related pain recurrence same characteristic, location and intensity, but less frequent (1-2 times per week), requiring Tramadol 200mg/day.
According to the patient, there was an improvement of $60 \%$ of the pain after the procedure.

DISCUSSION: Celiac plexus blocks with local anesthetics have been carried out for diagnostic purposes, but also as the primary treatment of pain in the chronic pancreatitis when associated with the use of corticosteroids. Neurolytic blockages of the plexus celiac are made with alcohol or phenol, but this modality is controversial as treatment of non-oncologic pancreatic pain, especially for the apparently short duration of the effect, which requires the repetition of the every 2 to 6 months.

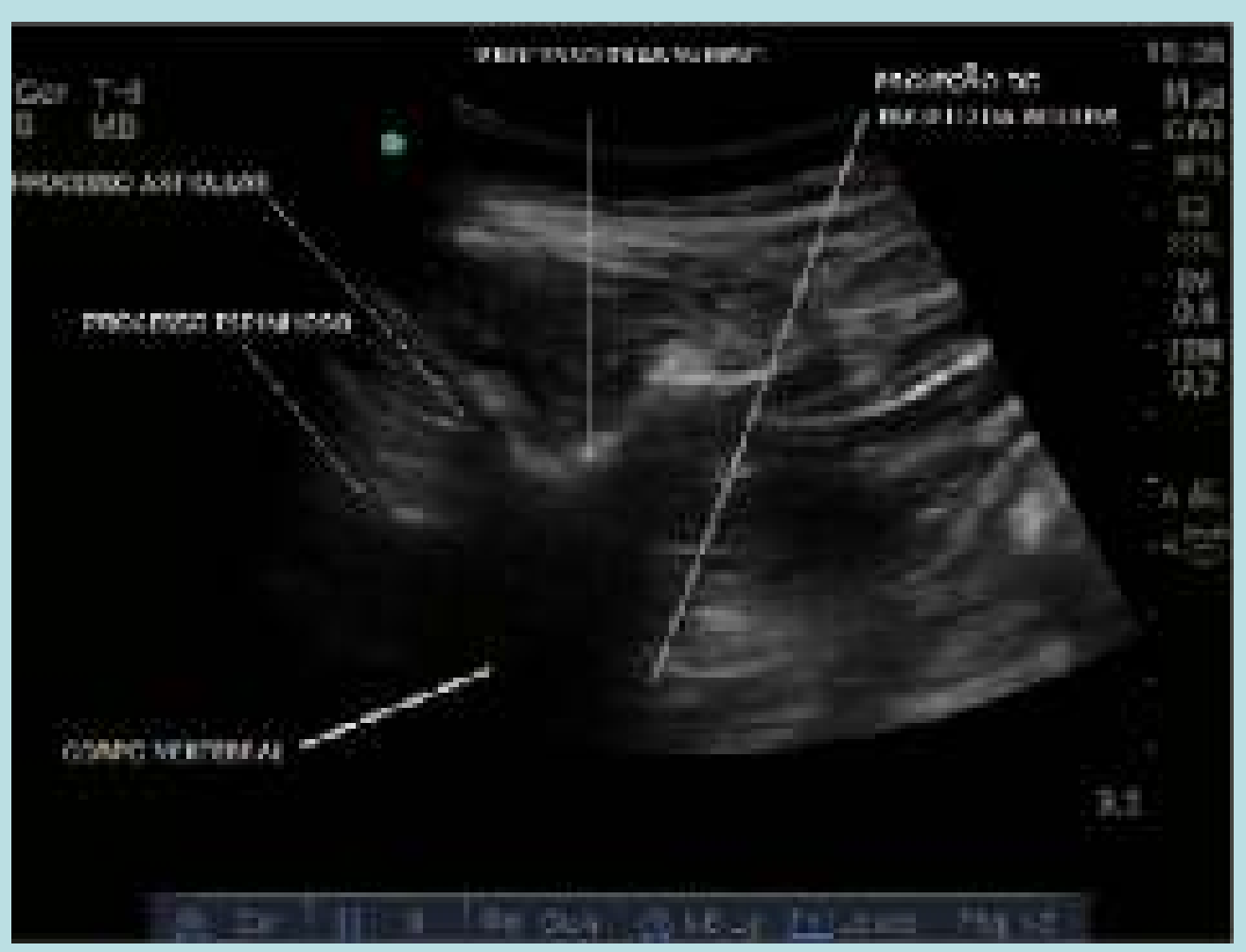

LEARNING POINTS: The use of botulinum toxin for the control of pain in is already established and is now an important possibility to be considered as an alternative therapy in difficult-to-control painful conditions, need further studies to support their effectiveness and safety.

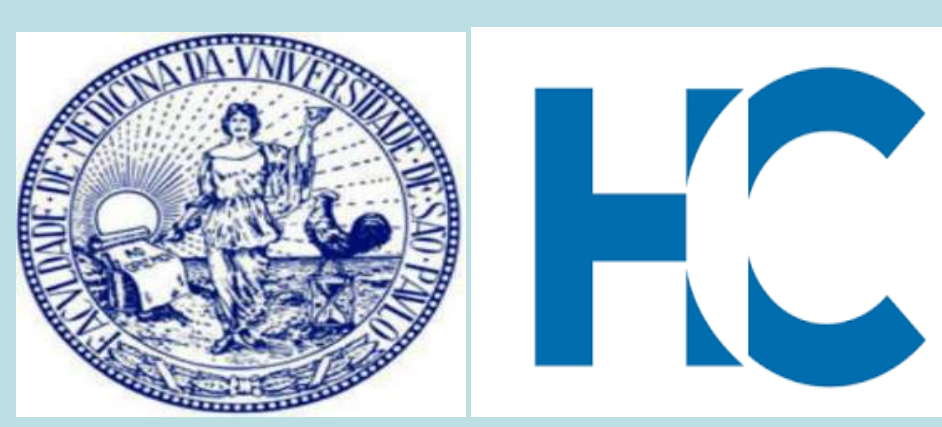

IOSPITAL DAS GLIINICAS DA FACULDADE DE MIEDICINIA 